\title{
ON NUMERICAL ENTROPY INEQUALITIES FOR A CLASS OF RELAXED SCHEMES
}

BY

HUAZHONG TANG (State key Laboratory of Scientific and Engineering Computing, Institute of Computational Mathematics, Academia Sinica, Beijing 100080, P. R. China),

TAO TANG (Dept. of Mathematics, Hong Kong Baptist University, Kowloon Tong, Hong Kong),

AND

JINGHUA WANG (Institute of Systems Science, Academia Sinica, Beijing 100080, P. R. China)

Abstract. In [4], Jin and Xin developed a class of first- and second-order relaxing schemes for nonlinear conservation laws. They also obtained the relaxed schemes for conservation laws by using a Hilbert expansion for the relaxing schemes. The relaxed schemes were proved to be total variational diminishing (TVD) in the zero relaxation limit for scalar equations. In this paper, by properly choosing the numerical entropy flux, we show that the relaxed schemes also satisfy the entropy inequalities. As a consequence, the $L^{1}$ convergence rate of $\mathcal{O}(\sqrt{\Delta t})$ for the relaxed schemes can be established.

1. Introduction. Consider the following stiff relaxation system:

$$
\begin{aligned}
\frac{\partial u}{\partial t}+\frac{\partial v}{\partial x} & =0 \\
\frac{\partial v}{\partial t}+a \frac{\partial u}{\partial x} & =-\frac{1}{\varepsilon}(v-f(u)) .
\end{aligned}
$$

The above system can be regarded as a singular perturbation problem, and the solutions are expected to converge, as $\varepsilon$ tends to zero, to the entropy solutions of the equilibrium

Received August 6, 1999.

2000 Mathematics Subject Classification. Primary 65M06.

Key words and phrases. Relaxing schemes, relaxed schemes, numerical entropy inequality, hyperbolic conservation laws.

The research of the first author was supported by the National Natural Science Foundation of China and the foundation of National key Laboratory of Computational Physics.

The research of the second author was supported by Hong Kong Baptist University.

The research of the third author was supported in part by the National Natural Science Foundation of China, and in part by NSF of US and NSF of China Joint Research Proposal No. INT-9601376.

E-mail address: thz@lsec.cc.ac.cn

E-mail address: ttang@math.hkbu.edu.hk

E-mail address: jwang@iss06.iss.ac.cn

(C)2001 Brown University 
equation

$$
\frac{\partial u}{\partial t}+\frac{\partial f(u)}{\partial x}=0
$$

There have been many recent studies concerning the asymptotic convergence of the relaxation systems to the corresponding equilibrium conservation laws as the rate of the relaxation tends to zero. Katsoulakis and Tzavaras [5] established an $\mathcal{O}(\sqrt{\varepsilon})$ error bound in the case that the equilibrium equation is a scalar multi-dimensional one. Kurganov and Tadmor [6] studied convergence and error estimates for a class of relaxation systems, including (1.1) and the one arising in chromatography, and concluded an $\mathcal{O}(\varepsilon)$ order of convergence for scalar convex conservation laws. For the relaxation system (1.1), Natalini [8] proved that the solutions to the relaxation system converge strongly to the unique entropy solution of (1.2) as $\varepsilon \rightarrow 0$. Teng established the first-order rate of convergence for (1.1) in the case when its equilibrium solutions are piecewise smooth [13], which is an improvement on the $\mathcal{O}(\sqrt{\varepsilon})$ error bounds $[5,6]$. The convergence and the rate of convergence mentioned above are mostly in the $L^{1}$ sense. In [12], Tadmor and Tang obtained the first-order pointwise rate of convergence for (1.1) in the case when its equilibrium solutions are piecewise smooth.

The relaxation system (1.1) is also a prototype of a class of nonoscillatory numerical schemes for systems of conservation laws. The linear hyperbolic structure of system (1.1) enables one to avoid the time-con-uming Riemann solvers, which are loaded by standard high resolution methods for nonlinear hyperbolic systems; proper implicit time discretizations can be taken to overcome the stability constraints brought in by possible stiffness. A finite speed of propagation is maintained. Moreover, the lower-order source term that is introduced by relaxation is not fully ranked and linear in the artificially introduced variable. Therefore, solving nonlinear systems of algebraic equations can be avoided. In [4], Jin and Xin developed a class of first- and second-order nonoscillatory numerical schemes for the conservation law (1.2), based on the local relaxation approximation (1.1).

The second-order relaxing scheme of the following form was proposed in [4]:

$$
\begin{aligned}
\frac{u_{j}^{n+1, \varepsilon}-u_{j}^{n, \varepsilon}}{\Delta t}+\frac{1}{2 \Delta x}\left(v_{j+1}^{n, \varepsilon}-v_{j-1}^{n, \varepsilon}\right) & -\frac{\sqrt{a}}{2 \Delta x}\left(u_{j+1}^{n, \varepsilon}-2 u_{j}^{n, \varepsilon}+u_{j-1}^{n, \varepsilon}\right) \\
+ & \frac{1-\beta}{4}\left[\left(\sigma_{j}^{+, \varepsilon}-\sigma_{j-1}^{+, \varepsilon}\right)-\left(\sigma_{j+1}^{-, \varepsilon}-\sigma_{j}^{-, \varepsilon}\right)\right]=0
\end{aligned}
$$

$$
\begin{array}{r}
\frac{v_{j}^{n+1, \varepsilon}-v_{j}^{n, \varepsilon}}{\Delta t}+\frac{a}{2 \Delta x}\left(u_{j+1}^{n, \varepsilon}-u_{j-1}^{n, \varepsilon}\right)-\frac{\sqrt{a}}{2 \Delta x}\left(v_{j+1}^{n, \varepsilon}-2 v_{j}^{n, \varepsilon}+v_{j-1}^{n, \varepsilon}\right) \\
+\frac{\sqrt{a}(1-\beta)}{4}\left[\left(\sigma_{j}^{+, \varepsilon}-\sigma_{j-1}^{+, \varepsilon}\right)+\left(\sigma_{j+1}^{-, \varepsilon}-\sigma_{j}^{-, \varepsilon}\right)\right] \\
=-\frac{1}{\varepsilon}\left(v_{j}^{n+1, \varepsilon}-f\left(u_{j}^{n+1, \varepsilon}\right)\right),
\end{array}
$$


where $\sigma_{j}^{ \pm, \varepsilon}$ and $\theta_{j}^{ \pm, \varepsilon}$ are defined by

$$
\begin{aligned}
\sigma_{j}^{ \pm, \varepsilon} & =\frac{1}{\Delta x} \Delta_{+}\left(v_{j}^{n, \varepsilon} \pm \sqrt{a} u_{j}^{n, \varepsilon}\right) \phi\left(\theta_{j}^{ \pm, \varepsilon}\right), \\
\theta_{j}^{ \pm, \varepsilon} & =\frac{\Delta_{-}\left(v_{j}^{n, \varepsilon} \pm \sqrt{a} u_{j}^{n, \varepsilon}\right)}{\Delta_{+}\left(v_{j}^{n, \varepsilon} \pm \sqrt{a} u_{j}^{n, \varepsilon}\right)}
\end{aligned}
$$

and $\beta=\mu=\sqrt{a} \frac{\Delta t}{\Delta x}, \Delta_{ \pm} u_{j}=\mp\left(u_{j}-u_{j \pm 1}\right)$ and $\left(u_{j}^{n, \varepsilon}, v_{j}^{n, \varepsilon}\right)$ is the approximation for the solutions of $(1.1)$ at $(j \Delta x, n \Delta t) . \phi(\theta)$ is a limiter function satisfying

$$
0 \leq \frac{\phi(\theta)}{\theta} \leq 2, \quad 0 \leq \phi(\theta) \leq 2
$$

Convergence theory for the second-order relaxing schemes (1.3)-(1.4) was provided by Aregba-Driollet and Natalini [1] and Wang and Warnecke [14]. Convergence rates for the corresponding first-order relaxing schemes, i.e., (1.3)-(1.4) with $\phi(\theta) \equiv 0$, were studied by Liu and Warnecke [7] and Yong [15]. Tang and Wu [11] studied the cell entropy inequalities for the relaxing schemes. Consult [9] for a bird's eye view of recent development on hyperbolic relaxation problems.

Since the leading-order behavior of relaxing schemes is governed by relaxed schemes when $\varepsilon$ is sufficiently small, it is important to study the behavior of the relaxed schemes. Using a Hilbert expansion gives the leading-order equations (as $\varepsilon \rightarrow 0^{n}$ ), i.e., the relaxed schemes for scalar conservation laws (1.2):

$$
\begin{aligned}
& v_{j}^{n}=f\left(u_{j}^{n}\right) \triangleq f_{j}^{n}, \\
& \frac{u_{j}^{n+1}-u_{j}^{n}}{\Delta t}+\frac{1}{2 \Delta x}\left(v_{j+1}^{n}-v_{j-1}^{n}\right)-\frac{\sqrt{a}}{2 \Delta x}\left(u_{j+1}^{n}-2 u_{j}^{n}+u_{j-1}^{n}\right) \\
& \quad+\frac{1-\beta}{4}\left[\left(\sigma_{j}^{+}-\sigma_{j-1}^{+}\right)-\left(\sigma_{j+1}^{-}-\sigma_{j}^{-}\right)\right]=0 .
\end{aligned}
$$

It can be shown that the above relaxed schemes are consistent and stable discretizations of the original conservation laws (1.2). Jin and Xin [4] also proved that they are TVD for the scalar conservation laws, thus are non-oscillatory and converge to the weak solutions of (1.2). To obtain the TVD property, the following assumptions are used in [4]:

$$
\begin{array}{ll}
\sqrt{a} \frac{\Delta t}{\Delta x}=\mu<1 ; & \text { CFL condition } \\
\sup _{u}\left|f^{\prime}(u)\right| \leq \sqrt{a} ; & \text { subcharacteristic condition } \\
0 \leq \frac{\phi(\theta)}{\theta} \leq 2, \quad 0 \leq \phi(\theta) \leq 2, & \text { limiter function condition. }
\end{array}
$$

The main objective of this work is to provide a rigorous proof of the cell entropy inequality for the relaxed schemes (1.7). The cell entropy inequality will be obtained 
under slightly stronger conditions:

$$
\begin{array}{ll}
\sqrt{a} \frac{\Delta t}{\Delta x}=\mu<1 ; & \text { CFL condition } \\
\sup _{u}\left|f^{\prime}(u)\right| \leq \frac{1}{\alpha} \sqrt{a} ; & \text { subcharacteristic condition } \\
0 \leq \frac{\phi(\theta)}{\theta} \leq X, \quad 0 \leq \phi(\theta) \leq X, & \text { limiter function condition. }
\end{array}
$$

The parameters $\alpha$ and $X$ in the conditions (1.8)-(1.10) satisfy

$$
\alpha>1, \quad 0<X<2, \quad 1-\frac{1}{\alpha} \geq X(1-\mu) .
$$

THEOREM 1. If the relaxed scheme (1.7) satisfies the assumptions (1.8)-(1.11), then the following cell entropy inequality holds for any entropy pair $(U, F)$, with convex $U$,

$$
\frac{U_{j}^{n+1}-U_{j}^{n}}{\Delta t}+\frac{1}{\Delta x}\left(\widehat{F}_{j+1 / 2}^{n}-\widehat{F}_{j-1 / 2}^{n}\right) \leq 0
$$

where the numerical entropy flux $\widehat{F}_{j+1 / 2}^{n}$ is defined by

$$
\begin{aligned}
\widehat{F}_{j+1 / 2}^{n}=\frac{1}{2}\left[F_{j+1}^{n}+F_{j}^{n}\right. & \left.-\sqrt{a}\left(U_{j+1}^{n}-U_{j}^{n}\right)\right] \\
& +\frac{(1-\mu)}{4}\left[\phi_{j}^{+} \Delta_{+}\left(F_{j}^{n}+\sqrt{a} U_{j}^{n}\right)-\frac{\phi_{j+1}^{-}}{\theta_{j+1}^{-}} \Delta_{+}\left(F_{j}^{n}-\sqrt{a} U_{j}^{n}\right)\right]
\end{aligned}
$$

where $U_{j}^{n}=U\left(u_{j}^{n}\right), F_{j}^{n}=F\left(u_{j}^{n}\right)$, and $\phi_{j}^{ \pm}=\phi\left(\theta_{j}^{ \pm}\right)$.

2. Proof of Theorem 1. This section is devoted to the proof of Theorem 1. Substituting $f_{j}^{n}$ for $v_{j}^{n}$ in the relaxed scheme (1.7) gives

$$
\begin{aligned}
\frac{u_{j}^{n+1}-u_{j}^{n}}{\Delta t}+ & {\left[\frac{1}{2 \Delta x}+\frac{1-\mu}{4 \Delta x}\left(\phi_{j}^{+}-\frac{\phi_{j+1}^{-}}{\theta_{j+1}^{-}}\right)\right]\left(f_{j+1}^{n}-f_{j}^{n}\right) } \\
& -\left[\frac{1}{2 \Delta x}+\frac{1-\mu}{4 \Delta x}\left(\phi_{j}^{+}+\frac{\phi_{j+1}^{-}}{\theta_{j+1}^{-}}\right)\right] \sqrt{a}\left(u_{j+1}^{n}-u_{j}^{n}\right) \\
+ & {\left[\frac{1}{2 \Delta x}-\frac{1-\mu}{4 \Delta x}\left(\phi_{j-1}^{+}-\frac{\phi_{j}^{-}}{\theta_{j}^{-}}\right)\right]\left(f_{j}^{n}-f_{j-1}^{n}\right) } \\
+ & {\left[\frac{1}{2 \Delta x}-\frac{1-\mu}{4 \Delta x}\left(\phi_{j-1}^{+}+\frac{\phi_{j}^{-}}{\theta_{j}^{-}}\right)\right] \sqrt{a}\left(u_{j}^{n}-u_{j-1}^{n}\right)=0 . }
\end{aligned}
$$


For any given entropy pair $(U, F)$ of $(1.2)$ with convex $U$, using integration by parts gives the following identity (see [2]):

$$
U^{\prime}(a)(f(b)-f(a))=F(b)-F(a)-\int_{a}^{b}(f(b)-f(s)) U^{\prime \prime}(s) d s
$$

where $F(b)=\int_{0}^{b} f^{\prime}(s) U^{\prime}(s) d s$. Using this identity, we can obtain

$$
\begin{aligned}
& U^{\prime}\left(u_{j}^{n}\right)\left(u_{j}^{n+1}-u_{j}^{n}\right)=U_{j}^{n+1}-U_{j}^{n}-\int_{u_{j}^{n}}^{u_{j}^{n+1}}\left(u_{j}^{n+1}-s\right) U^{\prime \prime}(s) d s \\
& U^{\prime}\left(u_{j}^{n}\right)\left(f_{j \pm 1}^{n}-f_{j}^{n}\right)=F_{j \pm 1}^{n}-F_{j}^{n}-\int_{u_{j}^{n}}^{u_{j \pm 1}^{n}}\left(f_{j \pm 1}^{n}-f(s)\right) U^{\prime \prime}(s) d s \\
& U^{\prime}\left(u_{j}^{n}\right)\left(u_{j \pm 1}^{n}-u_{j}^{n}\right)=U_{j \pm 1}^{n}-U_{j}^{n}-\int_{u_{j}^{n}}^{u_{j \pm 1}^{n}}\left(u_{j \pm 1}^{n}-s\right) U^{\prime \prime}(s) d s .
\end{aligned}
$$

We define $I_{1}$ as follows:

$$
I_{1}:=U^{\prime}\left(u_{j}^{n}\right) \cdot \text { LHS of }(2.1) .
$$

Using the identities (2.3) gives

$$
\begin{aligned}
I_{1}= & \frac{U_{j}^{n+1}-U_{j}^{n}}{\Delta t}-\frac{1}{\Delta t} \int_{u_{j}^{n}}^{u_{j}^{n+1}}\left(u_{j}^{n+1}-s\right) U^{\prime \prime}(s) d s \\
& +\left[\frac{1}{2 \Delta x}+\frac{1-\mu}{4 \Delta x}\left(\phi_{j}^{+}-\frac{\phi_{j+1}^{-}}{\theta_{j+1}^{-}}\right)\right]\left(F_{j+1}^{n}-F_{j}^{n}-\int_{u_{j}^{n}}^{u_{j+1}^{n}}\left(f_{j+1}^{n}-f(s)\right) U^{\prime \prime}(s) d s\right) \\
& -\left[\frac{1}{2 \Delta x}-\frac{1-\mu}{4 \Delta x}\left(\phi_{j}^{+}+\frac{\phi_{j+1}^{-}}{\theta_{j+1}^{-}}\right)\right] \sqrt{a}\left(U_{j+1}^{n}-U_{j}^{n}-\int_{u_{j}^{n}}^{u_{j+1}^{n}}\left(u_{j+1}^{n}-s\right) U^{\prime \prime}(s) d s\right) \\
& +\left[\frac{1}{2 \Delta x}-\frac{1-\mu}{4 \Delta x}\left(\phi_{j-1}^{+}-\frac{\phi_{j}^{-}}{\theta_{j}^{-}}\right)\right]\left(F_{j}^{n}-F_{j-1}^{n}+\int_{u_{j}^{n}}^{u_{j-1}^{n}}\left(f_{j-1}^{n}-f(s)\right) U^{\prime \prime}(s) d s\right) \\
& +\left[\frac{1}{2 \Delta x}-\frac{1-\mu}{4 \Delta x}\left(\phi_{j-1}^{+}+\frac{\phi_{j}^{-}}{\theta_{j}^{-}}\right)\right] \sqrt{a}\left(U_{j}^{n}-U_{j-1}^{n}+\int_{u_{j}^{n}}^{u_{j-1}^{n}}\left(u_{j-1}^{n}-s\right) U^{\prime \prime}(s) d s\right) .
\end{aligned}
$$


The right-hand side of (2.5) can be rewritten as

$$
\begin{aligned}
I_{1}= & \frac{U_{j}^{n+1}-U_{j}^{n}}{\Delta t}+\frac{1}{2 \Delta x}\left(F_{j+1}^{n}-F_{j-1}^{n}\right)-\frac{\sqrt{a}}{2 \Delta x}\left(U_{j+1}^{n}-2 U_{j}^{n}+U_{j-1}^{n}\right) \\
& +\frac{1-\mu}{4 \Delta x} \Delta_{-}\left[\phi_{j}^{+}\left(F_{j+1}^{n}+\sqrt{a} U_{j+1}^{n}-F_{j}^{n}-\sqrt{a} U_{j}^{n}\right)\right] \\
& -\frac{1-\mu}{4 \Delta x} \Delta_{-}\left[\frac{\phi_{j+1}^{-}}{\theta_{j+1}^{-}}\left(F_{j+1}^{n}-\sqrt{a} U_{j+1}^{n}-F_{j}^{n}+\sqrt{a} U_{j}^{n}\right)\right] \\
& -\frac{1}{\Delta t} \int_{u_{j}^{n}}^{u_{j}^{n+1}}\left(u_{j}^{n+1}-s\right) U^{\prime \prime}(s) d s \\
& -\left[\frac{1}{2 \Delta x}+\frac{1-\mu}{4 \Delta x}\left(\phi_{j}^{+}-\frac{\phi_{j+1}^{-}}{\theta_{j+1}^{-}}\right)\right] \int_{u_{j}^{n}}^{u_{j+1}^{n}}\left(f_{j+1}^{n}-f(s)\right) U^{\prime \prime}(s) d s \\
& +\left[\frac{1}{2 \Delta x}-\frac{1-\mu}{4 \Delta x}\left(\phi_{j}^{+}+\frac{\phi_{j+1}^{-}}{\theta_{j+1}^{-}}\right)\right] \sqrt{a} \int_{u_{j}^{n}}^{u_{j+1}^{n}}\left(u_{j+1}^{n}-s\right) U^{\prime \prime}(s) d s \\
& +\left[\frac{1}{2 \Delta x}-\frac{1-\mu}{4 \Delta x}\left(\phi_{j-1}^{+}-\frac{\phi_{j}^{-}}{\theta_{j}^{-}}\right)\right] \int_{u_{j}^{n}}^{u_{j-1}^{n}}\left(f_{j-1}^{n}-f(s)\right) U^{\prime \prime}(s) d s \\
& +\left[\frac{1}{2 \Delta x}-\frac{1-\mu}{4 \Delta x}\left(\phi_{j-1}^{+}+\frac{\phi_{j}^{-}}{\theta_{j}^{-}}\right)\right] \sqrt{a} \int_{u_{j}^{n}}^{u_{j-1}^{n}}\left(u_{j-1}^{n}-s\right) U^{\prime \prime}(s) d s .
\end{aligned}
$$

Denote the last five terms on the RHS of (2.6) by $I_{2}$. By changing the lower limit of the last four integrals from $u_{j}^{n}$ to $u_{j}^{n+1}$ we obtain

$$
\begin{aligned}
I_{2}= & \frac{1}{\Delta t} \int_{u_{j}^{n}}^{u_{j}^{n+1}} J_{0} U^{\prime \prime}(s) d s \\
& -\left[\frac{1}{2 \Delta x}+\frac{1-\mu}{4 \Delta x}\left(\phi_{j}^{+}-\frac{\phi_{j+1}^{-}}{\theta_{j+1}^{-}}\right)\right] \int_{u_{j}^{n+1}}^{u_{j+1}^{n}}\left(f_{j+1}^{n}-f(s)\right) U^{\prime \prime}(s) d s \\
& +\left[\frac{1}{2 \Delta x}-\frac{1-\mu}{4 \Delta x}\left(\phi_{j}^{+}+\frac{\phi_{j+1}^{-}}{\theta_{j+1}^{-}}\right)\right] \sqrt{a} \int_{u_{j}^{n+1}}^{u_{j+1}^{n}}\left(u_{j+1}^{n}-s\right) U^{\prime \prime}(s) d s \\
+ & {\left[\frac{1}{2 \Delta x}-\frac{1-\mu}{4 \Delta x}\left(\phi_{j-1}^{+}-\frac{\phi_{j}^{-}}{\theta_{j}^{-}}\right)\right] \int_{u_{j}^{n+1}}^{u_{j-1}^{n}}\left(f_{j-1}^{n}-f(s)\right) U^{\prime \prime}(s) d s } \\
+ & {\left[\frac{1}{2 \Delta x}-\frac{1-\mu}{4 \Delta x}\left(\phi_{j-1}^{+}+\frac{\phi_{j}^{-}}{\theta_{j}^{-}}\right)\right] \sqrt{a} \int_{u_{j}^{n+1}}^{u_{j-1}^{n}}\left(u_{j-1}^{n}-s\right) U^{\prime \prime}(s) d s }
\end{aligned}
$$


where $J_{0}$, which appears in the first integral of (2.7), is given by

$$
\begin{aligned}
J_{0}= & \left(u_{j}^{n+1}-s\right)-\Delta t \sqrt{a}\left[\frac{1}{2 \Delta x}-\frac{1-\mu}{4 \Delta x}\left(\phi_{j}^{+}+\frac{\phi_{j+1}^{-}}{\theta_{j+1}^{-}}\right)\right]\left(u_{j+1}^{n}-s\right) \\
& +\Delta t\left[\frac{1}{2 \Delta x}+\frac{1-\mu}{4 \Delta x}\left(\phi_{j}^{+}-\frac{\phi_{j+1}^{-}}{\theta_{j+1}^{-}}\right)\right]\left(f_{j+1}^{n}-f(s)\right) \\
& -\Delta t\left[\frac{1}{2 \Delta x}-\frac{1-\mu}{4 \Delta x}\left(\phi_{j-1}^{+}-\frac{\phi_{j}^{-}}{\theta_{j}^{-}}\right)\right]\left(f_{j-1}^{n}-f(s)\right) \\
& -\Delta t \sqrt{a}\left[\frac{1}{2 \Delta x}-\frac{1-\mu}{4 \Delta x}\left(\phi_{j-1}^{+}+\frac{\phi_{j}^{-}}{\theta_{j}^{-}}\right)\right]\left(u_{j-1}^{n}-s\right) .
\end{aligned}
$$

Multiply (2.1) by $U^{n}(s)$, integrate the resulting equation from $u_{j}^{n}$ to $u_{j}^{n+1}$, and then add the new resulting equation to Eq. (2.7). By the Mean-Value Theorem, we obtain

$$
\begin{aligned}
I_{2}= & \frac{1}{\Delta t} \int_{u_{j}^{n+1}}^{u_{j}^{n}}\left(u_{j}^{n}-s\right) J_{1} U^{\prime \prime}(s) d s+\int_{u_{j}^{n+1}}^{u_{j+1}^{n}}\left(u_{j+1}^{n}-s\right) J_{2} U^{\prime \prime}(s) d s \\
& +\int_{u_{j}^{n+1}}^{u_{j-1}^{n}}\left(u_{j-1}^{n}-s\right) J_{3} U^{\prime \prime}(s) d s .
\end{aligned}
$$

The functions $J_{1}, J_{2}$, and $J_{3}$ above are defined by

$$
\begin{aligned}
J_{1}= & (1-\mu)+\frac{\lambda(1-\mu)}{4}\left(\phi_{j-1}^{+}+\phi_{j}^{+}\right)\left(\sqrt{a}+f^{\prime}(\xi)\right) \\
& +\frac{\lambda(1-\mu)}{4}\left(\frac{\phi_{j+1}^{-}}{\theta_{j+1}^{-}}+\frac{\phi_{j}^{-}}{\theta_{j}^{-}}\right)\left(\sqrt{a}-f^{\prime}(\xi)\right), \\
J_{2}= & \sqrt{a}\left[\frac{1}{2 \Delta x}-\frac{1-\mu}{4 \Delta x}\left(\phi_{j}^{+}+\frac{\phi_{j+1}^{-}}{\theta_{j+1}^{-}}\right)\right]-f^{\prime}\left(\eta^{+}\right)\left[\frac{1}{2 \Delta x}+\frac{1-\mu}{4 \Delta x}\left(\phi_{j}^{+}-\frac{\phi_{j+1}^{-}}{\theta_{j+1}^{-}}\right)\right], \\
J_{3}= & \sqrt{a}\left[\frac{1}{2 \Delta x}-\frac{1-\mu}{4 \Delta x}\left(\phi_{j-1}^{+}+\frac{\phi_{j}^{-}}{\theta_{j}^{-}}\right)\right]+f^{\prime}\left(\eta^{-}\right)\left[\frac{1}{2 \Delta x}-\frac{1-\mu}{4 \Delta x}\left(\phi_{j-1}^{+}-\frac{\phi_{j}^{-}}{\theta_{j}^{-}}\right)\right],
\end{aligned}
$$

where $\xi \in\left(\min \left(u_{j}^{n+1}, u_{j}^{n}\right), \max \left(u_{j}^{n+1}, u_{j}^{n}\right)\right)$, and $\eta^{ \pm} \in\left(\min \left(u_{j}^{n+1}, u_{j+1}^{n}\right), \max \left(u_{j}^{n+1}, u_{j \pm 1}^{n}\right)\right)$ are some intermediate values, and $\lambda=\Delta t / \Delta x$. It follows from the assumption (1.11) that

$$
(\alpha-1) \geq \frac{1-\mu}{2}[(\alpha \pm 1) X+(\alpha \mp 1) X] \geq \frac{1-\mu}{2}\left[(\alpha \pm 1) \phi_{k}^{+}+(\alpha \mp 1) \frac{\phi_{k+1}^{-}}{\theta_{k+1}^{-}}\right]
$$

where $k=j$ or $j-1$. On the other hand, using the conditions (1.8)-(1.10) we obtain

$$
f^{\prime}\left(\eta^{+}\right)\left(1+\frac{1-\mu}{2}\left(\phi_{j}^{+}-\frac{\phi_{j+1}^{-}}{\theta_{j+1}^{-}}\right)\right) \leq \frac{\sqrt{a}}{\alpha}\left(1+\frac{1-\mu}{2}\left(\phi_{j}^{+}-\frac{\phi_{j+1}^{-}}{\theta_{j+1}^{-}}\right)\right)
$$


and

$$
-f^{\prime}\left(\eta^{-}\right)\left(1-\frac{1-\mu}{2}\left(\phi_{j}^{+}-\frac{\phi_{j+1}^{-}}{\theta_{j+1}^{-}}\right)\right) \leq \frac{\sqrt{a}}{\alpha}\left(1-\frac{1-\mu}{2}\left(\phi_{j}^{+}-\frac{\phi_{j+1}^{-}}{\theta_{j+1}^{-}}\right)\right)
$$

It follows from (2.12)-(2.14) that $J_{1}, J_{2}$, and $J_{3}$ defined by (2.9)-(2.11) are nonnegative. Using the inequality

$$
\int_{a}^{b}(b-s) h(s) d s \geq 0, \quad \text { if integrable function } h(s) \geq 0
$$

we obtain from $(2.8)$ that $I_{2} \geq 0$. Thus

$$
\begin{gathered}
\frac{U_{j}^{n+1}-U_{j}^{n}}{\Delta t}+\frac{1}{\Delta x}\left(\widehat{F}_{j+1 / 2}^{n}-\widehat{F}_{j-1 / 2}^{n}\right) \\
\quad=I_{1}-I_{2}=0-I_{2} \leq 0
\end{gathered}
$$

where $\widehat{F}_{j+1 / 2}^{n}$ is defined by (1.13). This completes the proof of Theorem 1 .

3. Remarks on convergence rate. In this work, we have provided a rigorous proof of the cell entropy inequalities (1.12). We now point out two immediate applications of Theorem 1:

- (i) The $\mathcal{O}(\sqrt{\Delta t})$ rate of $L^{1}$-convergence for the relaxed schemes (1.7) to entropy solution of scalar conservation law (1.2) can be established, based on the cell entropy inequalities (1.12) and the total variation boundedness given in [4].

- (ii) It follows from the results of Natalini [8], Wang and Warnecke [14], and Theorem 1 in this paper that numerical solutions of the second-order relaxing schemes (1.3)(1.4) converge to entropy solutions of scalar conservation laws as $\varepsilon \rightarrow 0^{+}$, and $\Delta x, \Delta t \rightarrow 0^{+}$, independent of the order of limits.

\section{REFERENCES}

[1] D. Aregba-Driollet and R. Natalini, Convergence of relaxation schemes for conservation laws, Appl. Anal. 61, 163-193 (1996)

[2] B. Cockburn and P.-A. Gremaud, A priori error estimates for numerical methods for scalar conservation laws. Part I: The general approach, Math. Comp. 65, 533-573 (1996)

[3] S. Jin, A convex entropy for a hyperbolic system with relaxation, J. Differential Equations 127, 95-107 (1996)

[4] S. Jin and Z.-P. Xin, The relaxing schemes for systems of conservation laws in arbitrary space dimensions, Comm. Pure Appl. Math. 48, 235-281 (1995)

[5] M. A. Katsoulakis and A. E. Tzavaras, Contractive relaxation systems and the scalar multidimensional conservation law, Comm. Partial Differential Equations 22, 195-233 (1997)

[6] A. Kurganov and E. Tadmor, Stiff systems of hyperbolic conservation laws, convergence and error estimates, SIAM J. Math. Anal. 28, 1446-1456 (1997)

[7] H. L. Liu and G. Warnecke, Convergence rates for relaxation schemes approximating conservation laws, SIAM J. Numer. Anal. 37, 1316-1337 (2000)

[8] R. Natalini, Convergence to equilibrium for the relaxation approximations of conservation laws, Comm. Pure Appl. Math. 49, 795-824 (1996)

[9] R. Natalini, Recent results on hyperbolic relaxation problems. Analysis of systems of conservation laws (Aachen, 1997), 128-198, Chapman and Hall/CRC Monogr. Surv. Pure Appl. Math., vol. 99, Chapman and Hall/CRC, Boca Raton, FL, 1999

[10] H. Nessyahu and E. Tadmor, Non-oscillatory central differencing for hyperbolic conservation laws, J. Comput. Phys. 87, 408-463 (1990) 
[11] H.-Z. Tang and H.-M. Wu, On a cell entropy inequality for the relaxing schemes of scalar conservation laws, J. Comput. Math. 18, 69-74 (2000)

[12] E. Tadmor and T. Tang, Pointwise error estimates for relaxation approximations to conservation laws, SIAM J. Appl. Math. (to appear)

[13] Z.-H. Teng, First-order $L^{1}$-convergence for relaxation approximations to conservation laws, Comm. Pure Appl. Math. 51, 857-895 (1998)

[14] J. Wang and G. Warnecke, Convergence of relaxing schemes for conservation laws, Advances in Nonlinear Partial Differential Equations and Related Areas, G.-Q. Chen, Y. Li, X. Zhu, and D. Cao (eds.), World Scientific Publishing, River Edge, NJ, 1998, pp. 300-325

[15] W. A. Yong, Numerical analysis of relaxation schemes for scalar conservation laws, Technical Report 95-30 (SFB 359), IWR, University of Heidelberg, 1995. 